This is an electronic version of an article published as: Penner, A.R. (2013). Induced energy polarization of the vacuum and the Coma cluster. Canadian Journal of Physics, 91(12), 1114-1120. DOI: 10.1139/cjp2013-0294

Canadian Journal of Physics is available online at: http://www.nrcresearchpress.com/journal/cjp and this article is available at: http://dx.doi.org/10.1139/cjp-2013-0294 


\title{
Induced energy polarization of the vacuum and the Coma cluster
}

Author:

\author{
A. Raymond Penner
}

Address:

Department of Physics,

Vancouver Island University, 900 Fifth Street, Nanaimo, BC, Canada, V9R 5S5

Email:_ raymond.penner@viu.ca

Tel: $\quad 250753-3245$ ext: 2336

Fax: $\quad 250740-6482$

PACS number: 90.95.30.-k 


\title{
Induced energy polarization of the vacuum and the Coma cluster
}

\begin{abstract}
The theory of an induced energy polarized vacuum is applied to the Coma cluster. The theoretical virial mass distribution of the cluster is determined and found to be in good agreement with previous virial mass estimates. A more concentrated intra-cluster gas profile than one based on the assumption that the gas is in hydrostatic equilibrium and isothermal does however lead to better agreement with measured shear values in the inner regions. The theory also leads to good agreement with measured velocity dispersion values in the case of the galaxies of the cluster being in radial orbits.
\end{abstract}

\section{Introduction}

The Coma cluster is one of the most studied of the rich galaxy clusters. Located at a distance of $100 \mathrm{Mpc}$ the cluster contains as many as 2000 galaxies as well as a large quantity of intra-galactic gas (ICG). Indeed, the mass of the ICG is estimated to be much greater than the total stellar mass residing in the galaxies. In addition to the ICG, gas is also found to be localized around two giant elliptical galaxies, NGC 4874, which is located at the centre of the cluster, and NGC 4889, which is at a projected distance of $300 \mathrm{kpc}$ from NGC 4874.

The first estimate of the total mass of the Coma cluster was provided by Zwicky [1]. Using the line-of-sight velocities and the positions of the cluster galaxies, Zwicky used the virial theorem to estimate that the total mass of the cluster would have to be at least 400 times larger than the observed luminous matter. Even with the large amount of ICG that was subsequently discovered, the virial mass of the Coma cluster is currently estimated to be an order of magnitude greater than the cluster's baryonic mass.

It is currently believed that dark matter provides the additional mass required to explain 
the observations of the Coma cluster. Simulations of the general formation of cold dark matter $(\mathrm{CDM})$ halos $[2,3]$ has shown that equilibrium density profiles of these halos is accurately fitted by the following NFW (Navarro-Frenk-White) profile,

$$
\rho_{\mathrm{NFW}}(\mathrm{r})=\frac{\rho_{\mathrm{SO}}}{\left(\frac{Y}{\mathrm{r}_{\mathbf{S}}}\right)\left(1+\frac{\mathrm{r}}{\mathrm{r}_{\mathrm{S}}}\right)^{2}}
$$

where $r_{\mathrm{s}}$ is a scale radius and $\rho_{\mathrm{so}}=\delta_{\mathrm{c}} \rho_{\text {crit }}$ where $\delta_{\mathrm{c}}$ is the characteristic overdensity of the halo in terms of the critical density, $\rho_{\text {crit }}$, required for closure. In dark matter theory the formation of the halos is independent of the baryonic mass. Baryonic mass, i.e. primordial gas, will of course fall into the potential wells of the halos leading to the formation of galactic clusters, but the existence of the halos in dark matter theory are independent of the baryonic mass.

The author has offered an alternative theory to dark matter [4]. In this theory the vacuum becomes polarized with respect to energy in the presence of a gravitational field. The vacuum is therefore analogous to a dielectric which becomes polarized with respect to charge in the presence of an electric field. The proposed theory is similar to the theory of dark matter in that the energy polarized vacuum provides a real energy or mass contribution to the gravitational field and therefore no modification of General Relativity or Newton's laws of motion are required. However, unlike dark matter theory, the contribution made to the gravitational field by the induced energy polarized vacuum is totally dependent on the baryonic mass and leads naturally to the baryonic Tully-Fisher relationship (BTFR) which relates the rotational speed of galaxies to their baryonic mass. The first application of the theory of an induced energy polarized vacuum was in the determination of the rotation curve for the Galaxy [5]. The theoretical rotation curve found was in excellent agreement with observation. The theory was also applied in general to the rotation curves of spiral galaxies [6]. It was found that the theory readily produced the features seen in observed rotation curves. The purpose of this manuscript is to now apply the theory of an induced energy polarized vacuum to a galaxy cluster, specifically the Coma cluster.

The outline of this paper is as follows. In section 2 the various methods that have been used to estimate the virial mass of the Coma cluster will be discussed. In section 3 the elements of the theory of an induced energy polarized vacuum required for the analysis of the Coma 
cluster will be presented. In addition, this section will provide a model of the baryonic mass distribution of the Coma cluster. In section 4, from the model of the baryonic mass distribution, the theoretical virial mass distribution of the Coma cluster will be generated and compared to the various virial mass estimates. In addition, the theoretical shear and velocity dispersion profiles for the Coma cluster will be determined and compared to observation. A conclusion follows in section 5. Throughout the paper a value of $\mathrm{H}_{\mathrm{o}}=70 \mathrm{~km} \mathrm{~s}^{-1} \mathrm{Mpc}^{-1}$ will be used.

\section{Virial mass determination}

There are three general methods that have been used to estimate the virial mass of the Coma cluster: X-ray observations of the ICG, galaxy kinematics, and gravitational lensing.

\subsection{X-ray observations}

The intra-cluster gas within the Coma cluster is a strong source of X-ray emission. Assuming hydrostatic equilibrium and spherical symmetry for the ICG it follows that

$$
\nabla \mathrm{P}=-\rho_{\text {gas }} \nabla \Phi
$$

where $\mathrm{P}(\mathrm{r})$ and pgas $(r)$ are respectively the radial gas pressure and gas density profiles and $\Phi(\mathrm{r})$ is the gravitational potential profile of the cluster. Using the ideal gas law, (2) can be written as the following differential equation for the gas temperature $\mathrm{T}(\mathrm{r})$,

$$
\frac{d T}{d r}=-\frac{1}{\rho_{g a s}} \frac{d p_{g a s}}{d r} T-\frac{G u m_{H}}{k} \frac{1}{r^{2}} M_{V}
$$

where $\mathrm{M}_{\mathrm{V}}(\mathrm{r})$, the virial mass distribution, is given by

$$
\mathrm{M}_{\mathrm{V}}=4 \pi \int_{0}^{r} \mathrm{r}^{\prime 2} \rho_{\mathrm{v}} \mathrm{d} \mathrm{r}^{\prime}
$$

where $\rho_{\mathrm{V}}\left(\mathrm{r}^{\prime}\right)$ is the virial density profile.

From analysis of the ROSAT X-ray all sky survey data Briel et al found [7] that a good fit was provided by the following X-ray luminosity profile: 


$$
\mathrm{S}=\mathrm{S}_{\mathrm{o}}\left(1+\left(\frac{\mathrm{r}}{\mathrm{r}_{\mathrm{g}}}\right)^{2}\right)^{-3 \beta_{\mathrm{g} z}+\frac{2}{2}}
$$

where $r_{c}=0.309 \mathrm{Mpc}, \beta_{\text {gas }}=0.75$, and $S_{o}$ is the central surface brightness. If the gas is treated as being isothermal then the radial gas density profile can be determined from the X-ray luminosity profile. Using (5) the gas density profile therefore follows [8]:

$$
\rho_{\mathrm{gas}}=\rho_{\mathrm{go}}\left(1+\left(\frac{\mathrm{g}}{\mathrm{r}_{\mathrm{c}}}\right)^{2}\right)^{-\frac{g_{0}}{2} \beta_{\mathrm{g} a z}} .
$$

where the central gas density is determined to be $\rho_{\mathrm{go}}=6.49 \times 10^{-24} \mathrm{~kg} \mathrm{~m}^{-3}$.

Estimating the virial mass of the Coma cluster from the X-ray data typically consists of assuming various forms for the virial mass profile and generating by (3) and (6) a temperature profile for the gas. This generated temperature profile is then compared to observations.

Hughes considered a wide range of virial mass profiles along with the constraint that the average gas temperature for the generated temperature profiles agreed with data from the Japanese X-ray satellite Tenma and the European satellite EXOSAT [9]. Analysis of the X-ray energy spectrum from Tenma and EXOSAT yielded an average $\mathrm{kT}$ of $(7.5 \pm 0.2) \mathrm{keV}$ and $(8.5$ $\pm 0.3) \mathrm{keV}$ respectively, over a radial extent of approximately $1.5 \mathrm{Mpc}$. Hughes found that a large class of dark matter distributions were consistent with the data, with the preferred model being one in which the virial mass distribution follows the distribution of the optical light (referred to as the mass-follows-light model). For this model the total mass contained within 3.57 Mpc was equal to $(1.3 \pm 0.2) \times 10^{15} \mathrm{M} \odot$. Hughes followed this up with analysis where the more constraining temperature profile as provided by the Ginga satellite was used [10]. The Ginga data indicates that the temperature of the gas is nearly constant at a temperature of $8 \mathrm{keV}$ out to approximately 1.2 Mpc after which it steadily falls out to the limits of the observations, i.e. at approximately 2.2 Mpc. For the virial mass profile distribution that was assumed in this analysis the virial mass contained within $3.57 \mathrm{Mpc}$ was estimated at $(1.4 \pm 0.6) \times 10^{15} \mathrm{M} \odot$, in good agreement with the previous estimate. The ROSAT all sky survey measured X-ray emissions out to $3 \mathrm{Mpc}$. Using this radial extent and the same average temperature constraint as used by Hughes, Briel et al found that for their assumed virial mass profiles that the total virial mass 
within $3.57 \mathrm{Mpc}$ was $(1.3 \pm 0.4) \times 10^{15} \mathrm{M} \odot$ [7]. Balland and Blanchard considered a wide range of virial mass profiles along with various temperature constraints [11]. For acceptable mass profiles they found that the enclosed virial mass within $3.57 \mathrm{Mpc}$ ranged from $0.93-6.6 \times 10^{15}$ $\mathrm{M} \odot$

One challenge with this technique of determining the virial mass of the Coma cluster is that in addition to the assumptions of the gas being in hydrostatic equilibrium and being isothermal, (3) is also very sensitive to boundary conditions. Indeed Balland and Blanchard found that differences as small as $0.5 \%$ in the central binding density values can lead to widely divergent temperature profiles.

\subsection{Kinematics}

This method follows Zwicky in that the measurements of the line-of-sight velocities and positions of the galaxies within the cluster are used to determine the cluster's virial mass. There are several different ways that these observations have been used. Kent and Gunn considered different distribution functions for the energy and the total angular momentum of cluster galaxies and generated theoretical velocity dispersion and surface density profiles for the galaxies [12]. These were then compared with observations. Models with distribution functions in which the ratio of tangential to radial velocity dispersions was constant gave acceptable results. Their best fitting model gave a virial mass within 5.30 Mpc of 2.07 x $10^{15} \mathrm{M} \odot$. Geller et al used the amplitude of the redshift caustics (the boundaries in the line-of-sight velocity vs. projected radius plots) to analyze the infall region of Coma [13]. From their results the virial mass of the Coma cluster within $5 \mathrm{Mpc}$ is estimated to be $(1.7 \pm 0.6) \times 10^{15} \mathrm{M} \odot$.

Other kinematic analyzes have used the following stellar hydrodynamic or Jeans equation for the spherical Coma cluster assuming the galaxies to be in equilibrium:

$$
\frac{\mathrm{d}}{\mathrm{dr}}\left(\mathrm{n}_{\mathrm{gal}} \sigma_{\mathrm{r}}^{2}\right)+2 \frac{\beta}{\mathrm{r}} \mathrm{n}_{\mathrm{gal}} \sigma_{\mathrm{r}}^{2}=-\mathrm{n}_{\mathrm{gal}} \frac{\mathrm{GM}_{\mathrm{V}}}{\mathrm{r}^{2}}
$$

where $n_{\text {gal }}(r)$ is the space density profile for the galaxies, $\sigma_{r}(r)$ is the radial component of the velocity dispersions, and $\beta(\mathrm{r})$, referred to as the velocity anisotropy parameter, is given by 


$$
\beta=1-\frac{\sigma_{\tau}^{2}}{\sigma_{\mathrm{r}}^{2}}
$$

where $\sigma_{\mathrm{t}}(\mathrm{r})$ is the tangential component of the velocity dispersions. For isotropic orbits $\sigma_{\mathrm{r}}=\sigma_{\mathrm{t}}$ and $\beta=0$, for circular orbits $\sigma_{\mathrm{r}}=0$ and $\beta \rightarrow-\infty$, and for radial orbits $\sigma_{\mathrm{t}}=0$ and $\beta=1$. The velocity dispersion components $\sigma_{\mathrm{r}}$ and $\sigma_{\mathrm{t}}$ are not however directly observed. What is observed are the line-of-sight velocity dispersions $\sigma_{\text {los }}(r)$. From (7) and by the geometry it can be shown [8] that

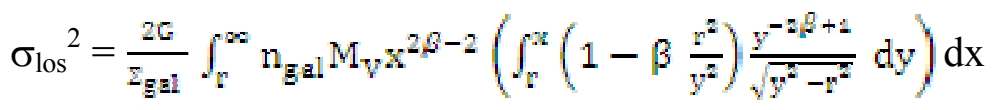

where $\Sigma_{\text {gal }}(\mathrm{r})$ is the surface density profile for the galaxies. The following surface density and space density (which is determined by deprojecting the $\Sigma_{\text {gal }}(\mathrm{r})$ ) profile, are provided for the Coma cluster galaxies [14];

$$
\begin{aligned}
& \Sigma_{\text {gal }}=\Sigma_{0}\left(1+\left(\frac{\mathrm{r}}{\mathrm{r}_{\mathrm{o}}}\right)^{2}\right)^{(1-\mathrm{w} / 2} \\
& \mathrm{n}_{\text {gal }}=\frac{\Sigma_{0}}{2.2 \mathrm{r}_{0}}\left(1+\left(\frac{\mathrm{r}}{\mathrm{r}_{0}}\right)^{2}\right)^{-\gamma / 2}
\end{aligned}
$$

where $\Sigma_{0}=1176$ galaxies $\operatorname{deg}^{-2}, \mathrm{r}_{0}=0.213 \mathrm{Mpc}$, and $\gamma=2.72$.

In (9) both $\Sigma_{\text {gal }}(r)$ and $\sigma_{\text {los }}(r)$ are determined observationally while the space density profile, $\mathrm{n}_{\mathrm{gal}}(\mathrm{r})$, is determined by deprojecting $\Sigma_{\mathrm{gal}}(\mathrm{r})$. Equation (9) is therefore degenerate in terms of the virial mass distribution $\mathrm{M}_{\mathrm{V}}(\mathrm{r})$ and the velocity anisotropy parameter $\beta(\mathrm{r})$. Analysis typically proceeds by assuming various forms for the virial mass distribution and testing whether the resulting velocity anisotropy is physically reasonable or by assuming various forms for both $M_{V}(r)$ and $\beta(r)$ and comparing the resulting $\sigma_{\text {los }}(r)$ with observation. In general, models where the mass is concentrated in the core of the cluster lead to relatively low virial masses and require the galaxies to be on near circular orbits, i.e. $\beta \rightarrow-\infty$. Models where the virial mass distribution is weakly concentrated lead to relatively high virial masses and require the galaxies to be on 
primarily radial orbits, i.e. $\beta=1$. Models where the virial mass distribution follows the galaxy distribution, i.e. mass-follows-light model, fall in between these two, and the resulting orbits are in general more isotropic, i.e. $\beta \cong 0$. The and White found from their mass models that the virial mass within $3.86 \mathrm{Mpc}$ of the cluster centre can range from $4.3 \times 10^{14} \mathrm{M} \odot$ to $3.6 \times 10^{15} \mathrm{M} \odot$ [14]. The model that was found to provide the best fit to the observed velocity dispersions was the mass-follows-light model where the virial mass within $3.86 \mathrm{Mpc}$ was $(1.4 \pm 0.4) \times 10^{15} \mathrm{M} \odot$. Merritt also found that a fairly wide range of distributions for $\mathrm{M}_{\mathrm{V}}(\mathrm{r})$ provided physically reasonable results [15]. However the enclosed virial mass within 1.43 Mpc obtained from their models was always close to 8.6 x $10^{14} \mathrm{M} \odot$. Lokas and Mamon considered several different possible virial mass distributions and determined the corresponding velocity dispersion and the kurtois profiles [8]. For their best fitting model the virial mass within $2.9 \mathrm{Mpc}$ was found to be $(1.4 \pm 0.4) \times 10^{15} \mathrm{M} \odot$.

\subsection{Weak gravitational lensing}

In this method the shape distortion of background galaxies caused by the gravitational field of the Coma cluster are used to determine the virial mass of the cluster. This technique is independent of any dynamical assumptions. The major issue with this technique is that since the Coma cluster is at such a low redshift using weak lensing becomes difficult since a large area surrounding the cluster requires imaging.

In general, the tangential shear, $\gamma(r)$, of background galaxies due to a foreground cluster lens is given by

$$
\gamma=\Sigma_{\mathrm{crit}}^{-1}\left(\frac{\mathrm{M}_{\mathrm{V}}}{\pi \mathrm{r}^{2}}-\Sigma_{\mathrm{V}}\right)
$$

where $\Sigma_{\mathrm{V}}(\mathrm{r})$ is the projected surface virial mass density (Miralda-Escude [16]) and $\Sigma_{\text {crit }}$ is the critical surface mass density. Kubo et al determined shear values for distances from the cluster centre ranging from approximately 1.7 to $14 \mathrm{Mpc}$ [17]. For their best fitting NFW virial mass density profile $\left(\rho_{\mathrm{so}}=9.29 \times 10^{-23} \mathrm{~kg} \mathrm{~m}^{-3}, \mathrm{r}_{\mathrm{s}}=0.518 \mathrm{Mpc}\right)$ the total enclosed mass within $2.84_{-0.3}^{+0.3}$ Mpc was found to be $2.69_{-0.8}^{+0.93} \times 10^{15} \mathrm{M} \odot$. Gavazzi et al determined shear values for distances 
from the cluster centre ranging from approximately 18 to $620 \mathrm{kpc}$ [18]. Their best fitting NFW

profile $\left(\rho_{\mathrm{so}}=6.15 \times 10^{-23} \mathrm{~kg} \mathrm{~m}^{-3}, \mathrm{r}_{\mathrm{s}}=0.360 \mathrm{Mpc}\right)$ results in an enclosed mass of $5.1_{-2: 1}^{+4.3} \times 10^{14} \mathrm{M} \odot$ within a radius of $1.8_{-0.5}^{+0.5} 10^{15} \mathrm{Mpc}$.

All above methods used in estimating the virial mass of the Coma cluster use assumed models for the virial mass distribution. The resulting theoretical velocity anisotropic parameter, gas temperature profile, or shear profiles are then fitted, through the use of free parameters in their models, to either observations or (in the case of the kinematic method) to expected behavior. In the theory of an induced energy polarized vacuum the virial mass distribution of a galaxy or a cluster is determined uniquely by the galaxy's or cluster's baryonic mass distribution. There are no free parameters in the theory to fit to observations. The need for assumptions on the form of the virial mass distribution is therefore eliminated. Section 3 will provide a summary of this theory along with a model of the baryonic mass distribution of the Coma cluster.

\section{Theory}

\subsection{Induced energy polarization of the vacuum}

In the theory of an induced energy polarized vacuum, entities in the vacuum become polarized in the presence of a gravitational field. From the specifics of the model the induced energy dipole moment density, $\mathbf{P}_{\mathbf{E}}$, along with its dependence on the gravitational field is then determined. Given this $\mathbf{P}_{\mathbf{E}}$ the mass density of the vacuum, $\rho_{\mathrm{E}}$, surrounding a given gravitational field source is then found by

$$
\rho_{\mathrm{E}}=-\frac{\nabla \cdot \mathrm{P}_{\mathrm{E}}}{c^{z}}
$$

It follows that the contribution to the gravitational field, $g_{v}(r)$, due to the energy polarized vacuum, will be given by

$$
\mathrm{g}_{\mathrm{V}}=\frac{\mathrm{G}}{\mathrm{r}^{2}} \int_{0}^{\mathrm{r}} \rho_{\mathrm{E}} \mathrm{dV}
$$

which in the case of spherical symmetry, by (13-14), results in 


$$
\mathrm{g}_{\mathrm{v}}=\frac{4 \pi \mathrm{G}}{\mathrm{c}^{2}} \mathrm{P}_{\mathrm{E}}
$$

The total gravitational field surrounding a given baryonic mass is then given by

$$
\mathrm{g}=\mathrm{g}_{\mathrm{B}}+\mathrm{g}_{\mathrm{v}}
$$

where $\mathrm{g}_{\mathrm{B}}$ is the gravitational field due to the baryonic mass distribution. As stated in the introduction the theory of an induced energy polarized vacuum is analogous to the case of a dielectric in the presence of an electric field. In that case it is electric dipole moments that are induced within the dielectric with an equation analogous to (13) used to determine the induced charge density within the dielectric. As shown by the author the theory of an induced energy polarized vacuum leads naturally to the BTFR [4].

The author has provided a semi-classical model of how the vacuum becomes polarized [4]. Although it is suspected that the entities in question are quantum fluctuations within the vacuum, it is believed by the author that the semi-classical model is a reasonable approximation. In the model it is hypothesized that throughout the cosmos entities of both net positive and net negative energy continually come into and out of existence with a maximum lifetime, $\tau$, as given by the Heisenberg uncertainty principle. In the presence of a gravitational field each entity will move during its' lifetime and subsequently each entity is equivalent to an energy dipole. A model of the effect of interactions results in the following dependence of the induced energy dipole moment density, $\mathbf{P}_{\mathbf{E}}(\mathrm{g})$, on the total gravitational field,

$$
\mathbf{P}_{\mathbf{E}}=\frac{\mathrm{c}^{2}}{4 \pi \mathrm{G}} \mathrm{g}_{0}\left[\frac{3}{2}\left(1-\frac{\mathrm{a}^{-\gamma\left(\frac{\mathrm{g}}{\mathrm{g}}\right)}}{\gamma}\right)\right] \mathrm{g}
$$

where

$$
\gamma=\frac{\sqrt{\pi}}{2} \frac{\operatorname{err}\left(\sqrt{\gamma\left(\frac{\mathrm{g}}{\mathrm{g}_{0}}\right)}\right)}{\sqrt{\gamma\left(\frac{\mathrm{g}}{\mathrm{g}_{0}}\right)}}
$$

The value of the single parameter $\mathrm{g}_{0}$ in $(17-18)$ is given by 


$$
\mathrm{g}_{0}=\frac{3}{2 \mathrm{GA}}
$$

where A is the coefficient of the BTFR with the BTFR expressed as

$$
\mathrm{M}_{\mathrm{B}}=\mathrm{A} \mathrm{v}
$$

with $\mathrm{M}_{\mathrm{B}}$ being the baryonic mass of a galaxy and $\mathrm{v}$ being the galaxy's rotational speed. Using a value of $\left(45 \pm 10 \mathrm{~km}^{-4} \mathrm{~s}^{4} \mathrm{M} \odot\right)$ for $\mathrm{A}$, as given by McGaugh and Wolf [19], then results, via (19), in

$$
\mathrm{g}_{0}=(1.0 \pm 0.2) \times 10^{-10} \mathrm{~m} \mathrm{~s}^{-2}
$$

Therefore in the theory of an induced energy polarized vacuum, given only the baryonic mass distribution and the coefficient of the BTFR, the contribution to the gravitational field provided by the vacuum can be determined. There are no free parameters in the theory.

\subsection{Baryonic mass distribution of the Coma cluster}

The following three contributors to the baryonic mass of the Coma cluster will be considered: the intra-galactic gas, the stellar mass contained in the galaxies, and the gas localized around the two giant elliptical galaxies NGC 4874 and NGC 4889.

The ICG is the dominant baryonic component of the Coma cluster. From the observations of the X-ray emissions the ICG distribution is found to be approximately spherically symmetric about the cluster centre. If the gas is taken to be isothermal the gas density profile will be given by (6). If this profile is taken to be valid out to $3 \mathrm{Mpc}$, corresponding to the radial extent that Xray emission has been detected, the total ICG mass within $3 \mathrm{Mpc}$, using the values as given for (5-6), is $2.0 \times 10^{14} \mathrm{M} \odot$. If the gas density profile as given by (6) is valid out to $6 \mathrm{Mpc}$ the total ICG mass within $6 \mathrm{Mpc}$ is calculated to be $5.8 \times 10^{14} \mathrm{M} \odot$. At best these ICG mass estimates must be taken as a rough approximation as the GINGA results indicate that the gas is only approximately isothermal out to $1.2 \mathrm{Mpc}$. 
The gas profile as given by (6), based on the assumptions of the gas being isothermal as well as being in hydrostatic equilibrium and with the values as given for (5-6), will be referred to as the isothermal profile. In order to demonstrate the effect that the baryonic mass distribution has on the results, the case where the ICG is more concentrated with $\beta_{\text {gas }}$ in (6) being $50 \%$ greater, i.e $\beta_{\mathrm{g}}=1.125$, will also be considered in the analysis. The value for $r_{c}$ will be as given for the isothermal profile but the value for $\rho_{\mathrm{go}}$ is increased to $2.86 \times 10^{-23} \mathrm{~kg} \mathrm{~m}^{-3}$. This will result in the more concentrated profile containing the same amount of ICG within $3 \mathrm{Mpc}$ as the isothermal profile, i.e. $2.0 \times 10^{14} \mathrm{M} \odot$.

After the ICG the second most important contributors to the baryonic mass of the Coma cluster are the stars within the cluster's galaxies. Lokas and Mamon fitted a NFW profile to the surface luminosity data for the Coma cluster [8], and for their estimated mean mass-to-light ratio of $6.43 \mathrm{M} \odot / \mathrm{L}_{\odot}$ the following stellar mass density profile, $\rho_{\text {stars }}(\mathrm{r})$ then follows;

$$
\rho_{\text {stars }}=\frac{\rho_{30}}{\left(\frac{r}{r_{S}}\right)\left(1+\frac{r}{r_{3}}\right)^{2}}
$$

where $\rho_{\mathrm{so}}=1.54 \times 10^{-24} \mathrm{~kg} \mathrm{~m}^{-3}$ and $\mathrm{r}_{\mathrm{s}}=0.424 \mathrm{Mpc}$. This distribution results in a total stellar mass of $2.6 \times 10^{13} \mathrm{M} \odot$ within $3 \mathrm{Mpc}$ and a total stellar mass of $3.9 \times 10^{13} \mathrm{M} \odot$ within $6 \mathrm{Mpc}$. The total stellar mass of the galaxies is therefore approximately one order of magnitude less than the estimated ICG mass.

The third baryonic mass component that is considered is the gas that is localized around the two giant ellipticals, NGC 4874 and NGC 4889. From measurements of the sub clustering around the two ellipticals [20], Mellier et al estimated that the virial mass within $70 \mathrm{kpc}$ of NGC 4874 and NGC 4889 is $4.1 \times 10^{13} \mathrm{M} \odot$ and $5.4 \times 10^{13} \mathrm{M} \odot$ respectively. Of course this is the virial mass and the gas mass localized at these galaxies will be considerably less. For the analysis presented in this manuscript the total additional gas mass concentrated around both NGC 4874 and NGC 4889 will be taken to be $1.5 \times 10^{13} \mathrm{M} \odot$, which is approximately $16 \%$ of the total estimated virial mass of the two galaxies. As will be seen this value leads to good agreement with the shear measurements in the inner regions of the cluster. This value is also in reasonable agreement with the X-ray luminosities of NGC 4874 and NGC 4889 as given by Vikhilin, 
Forman and Jones [21]. From their measurements it is estimated that the total X-ray luminosities of these two ellipticals is approximately $9 \%$ of the total X-ray luminosity of the cluster. Given that the total ICG mass within $3 \mathrm{Mpc}$, the extent of observed X-ray emission, was estimated at $2.0 \times 10^{14} \mathrm{M}_{\odot}$, a value of $1.5 \times 10^{13} \mathrm{M}_{\odot}$ for the amount of gas concentrated around NGC 4874 and NGC 4889 is reasonable.

As an approximation and so as to be able to use (15), the $1.5 \times 10^{13} \mathrm{M} \odot$ of additional gas will be taken to be distributed symmetrically around the centre of the cluster, which is taken to be at the location of NGC 4874, with the following Gauss profile

$$
\rho_{\text {gas } 2}(r)=\frac{\rho_{\text {goz }} e^{-\frac{1}{2}\left(\frac{r}{\sigma}\right)^{2}}}{\left(\frac{r}{\sigma}\right)^{2}}
$$

where $\rho_{\mathrm{go} 2}=1.92 \times 10^{-23} \mathrm{~kg} \mathrm{~m}^{-3}$ and the standard deviation $\sigma$ is set at $150 \mathrm{kpc}$. This distribution will result in $95 \%$ of the additional $1.5 \times 10^{13} \mathrm{M} \odot$ of gas being located to within $300 \mathrm{kpc}$ of the cluster's centre which corresponds to the projected distance of NGC 4889. This is a crude approximation but as will be seen it will lead to good agreement with the shear measurements in the inner regions of the cluster.

Figure 1 shows the mass density profiles for the three baryonic components as well as the overall baryonic density profile as given by

$$
\rho_{\mathrm{B}}=\rho_{\text {stars }}+\rho_{\text {gas }}+\rho_{\text {gas } 2}
$$

for both the isothermal and the more concentrated ICG profiles. As is seen, beyond $200 \mathrm{kpc}$, for both profiles, the ICG is the dominant contributor to the baryonic mass of the Coma cluster. These two baryonic mass distributions will now be used in the theory of an induced energy polarized vacuum to determine the virial mass distribution of the Coma cluster.

\section{Results}

The method of obtaining the overall gravitational field of the Coma cluster is as follows. The initial estimate of the gravitational field is taken to be solely that due to the cluster's baryonic mass. The value of $\mathbf{P}_{\mathbf{E}}$ is then determined by (17) and the gravitational field due to the 


\section{Penner - Induced energy polarization of the vacuum and the Coma cluster}

energy polarized vacuum is then determined from (15). For the next estimate, the total gravitational field is taken to be equal to the sum of the fields due to both the baryonic mass and the polarized vacuum. This iterative process is then repeated until the resulting values of the total gravitational field obtained after a given iteration vary by less than $1 \%$ from the previous iteration.

Figure 2 show the two baryonic density profiles and the resulting theoretical virial density profiles for the Coma cluster. As is seen the differences between the isothermal and the more concentrated baryonic distributions are suppressed when it comes to the determination of the virial distributions. This is a general result of the theory of an induced energy polarized vacuum. Figure 3 shows the theoretical virial density profiles along with the dark matter NFW density profiles of Kubo et al and Gavazzi et al. The virial density profile that results from the theory of an induced energy polarized vacuum falls approximately within the same range as the NFW density profiles. Again, it must be stressed that the theoretical virial density profiles do not involve any free parameters. The theoretical virial density profiles result directly from the models of the baryonic mass distribution of the cluster.

One of the differences between the density profiles shown on Figure 3 are their slopes in

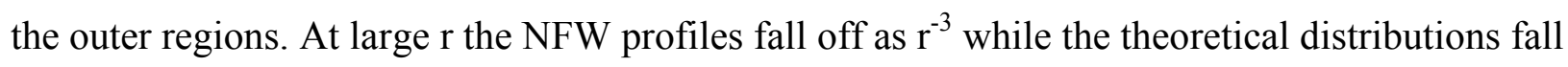
off approximately as $\mathrm{r}^{-1.5}$ for the isothermal profile and as $\mathrm{r}^{-2}$ for the more concentrated gas profile. The slope of the theoretical virial density profile at large $r$ will of course depend on the baryonic mass distribution but in general it will not fall off faster than $\mathrm{r}^{-2}$. This is again a general result of the theory of an induced energy polarized vacuum.

Figure 4 shows the theoretical virial mass distributions along with their associated baryonic mass distributions. As is seen the baryonic mass of the cluster only contributes approximately $10 \%$ of the virial mass of the cluster. Also included on Figure 4 are the various estimates of the virial mass of the Coma cluster that were provided in Section 2. As is seen, the theoretically determined virial mass distributions are overall consistent with these previous estimates. 
Given the determined virial mass distribution, the theoretical shear for the Coma cluster can be determined from (12). The result is shown on Figure 5 along with the shear measurements of both Kubo et al and Gavazzi et al. As is seen the simplified model of the gas contributions of NGC 4874 and NGC 4889 leads to good agreement with the shear measurements within $200 \mathrm{kpc}$. Beyond this distance is where differences between the two baryonic profiles become evident with the more concentrated ICG profile being in better agreement with the shear measurements of Gavazzi et al. The total amount of baryonic mass that has been estimated from observations does, via the theory of an induced energy polarized vacuum, therefore lead to good agreement with the shear measurements. The theoretical shear is however, for both profiles, less than the shear values obtained by Kubo et al. To obtain good agreement with these values the amount of baryonic mass contained within the Coma cluster would need to be greater than what is currently estimated.

Given the theoretical virial mass distribution, $\mathrm{M}_{\mathrm{v}}(\mathrm{r}),(9)$ is no longer degenerate and $\sigma_{\text {los }}(r)$ will therefore just be dependent on $\beta$, the velocity anisotropy parameter. The resulting theoretical line-of-sight velocity dispersions are shown on Figure 6 for $\beta=0$, corresponding to isotropic orbits, $\beta \rightarrow-\infty$, corresponding to circular orbits, and $\beta=1$, corresponding to radial orbits. Also included on this figure are the measured velocity dispersions as provided by Merritt [15] and by Lokas and Mamon [8]. From the figure it is seen that, independent of which of the two baryonic mass distributions is considered, the theoretical velocity dispersions in the case of radial orbits are in the best agreement with observation. Although, within $1.5 \mathrm{Mpc}$ of the cluster centre, for the concentrated baryonic mass distribution isotropic orbits also provide a reasonable fit.

\section{Conclusion}

The theory of an induced energy polarized vacuum using the baryonic mass distribution of the Coma cluster estimated from various observations is in good agreement with previous virial mass estimates. A more concentrated ICG profile than one based on the assumptions of gas being in hydrostatic equilibrium and being isothermal does however lead to better agreement with measured shear values in the inner region. The theory also leads to good agreement with measured velocity dispersions in the case of the galaxies of the cluster being in radial orbits. 
The virial density profile that results from the theory gives density values that are in the same range as those of the NFW profiles that have previously been fitted to two sets of shear measurements. However, it must again be stressed that, unlike the NFW profiles, the induced energy polarized vacuum theory contains no free parameters. Whether it be individual galaxies $([5,6])$ or a cluster such as Coma, the only inputs required by the theory are the baryonic mass distribution and the coefficient of the BTFR; with these inputs alone the total gravitational field of a galaxy or of a cluster is uniquely determined.

It follows from the theory of an induced energy polarized vacuum that the BTFR should in principle be applicable to galactic clusters as it is to spiral galaxies. However, the conditions required for the BTFR, namely a concentrated baryonic mass and outer circular orbits, do not apply in the case of the Coma cluster. For example, consider the more concentrated baryonic mass model. At a distance of $6 \mathrm{Mpc}$ from the clusters centre the total enclosed baryonic mass is $2.93 \times 10^{14}$ M॰. From the BTFR, as given by (20), the circular orbital velocity should be 1597 $\mathrm{km} / \mathrm{s}$. The relationship between circular velocity and the line-of-sight velocity dispersion when the rotational curve is flat (ref. [22]) is given by $\sigma_{\text {los }}=2^{-1 / 2} \mathrm{v}_{\text {circ. }}$ Therefore the line-of-sightvelocity dispersion should be $1129 \mathrm{~km} / \mathrm{s}$. From Figure 6, for the concentrated baryonic mass distribution and circular orbits, the theoretical line-of-sight-velocity dispersion at $6 \mathrm{Mpc}$ is 903 $\mathrm{km} / \mathrm{s}$. The two values are not that far off and using the BTFR for the cluster would seem to provide an approximation. However this approximation would only be valid if the galaxy orbits are circular. As shown in Figure 6, the outer orbits are radial and the observed line-of-sightvelocity dispersion value is approximately half of that corresponding to circular orbits.

The model of how the vacuum becomes polarized in a gravitational field as presented by the author [4] may seem somewhat ad hoc; however the semi-classical model should be viewed only as an approximation to a final theory. That being said, with regards to applications the model provided is quite adequate. Whether the rotational curves of galaxies or the dynamics of a cluster are considered, both fall into the realm of weak fields, which in terms of the theory corresponds to $\mathrm{g}<<\mathrm{g}_{0}$ with $\mathrm{g}_{0}$ as given by (21). In this weak field realm, almost any model based on the vacuum becoming energy polarized in a gravitational field will lead to basically the same results. The key feature required is that the relationship between the induced energy dipole moment density, $\mathbf{P}_{\mathbf{E}}$, and the total gravitational field, $\mathbf{g}$, is nonlinear [4,23]. 
Future work will be undertaken along two paths. One path will be to apply the theory to other galaxies and other clusters in order to provide a preponderance of evidence in support of the theory of an induced energy polarized vacuum. The second path will consider situations where the theory will diverge from standard dark matter theory. It is expected that one promising area will be in the case of binary galaxies.

\section{References}

1. F. Zwicky, Helv. Phys. Acta 6, 110 (1933)

2. J.F. Navarro, C.S. Frenk, and S.D.M. White. ApJ 462, 563 (1996)

3. J.F. Navarro, C.S. Frenk, and S.D.M. White. ApJ 490, 493 (1997)

4. A.R. Penner, Can. J. Phys. 90(4), 315 (2012)

5. A.R. Penner, Can. J. Phys. 91(2), 126 (2013)

6. A.R. Penner, Can. J. Phys. TBD (2013)

7. U.G. Briel, J.P. Henry, and H. Bohringer, A\&A 259, L31 (1992)

8. E.L. Lokas and G.A. Mamon, MNRAS 343(2), 401 (2003)

9. J.P. Hughes, ApJ 337, 21 (1989)

10. J.P. Hughes, arXiv:astro-ph/9709272v1 (1997)

11. C. Balland and A. Blanchard, arXiv:astro-ph/9510130 (1995)

12. S.M. Kent and J.E. Gunn, AJ 87, 945 (1982)

13. M.J. Geller, A. Diaferio, and M.J. Kurtz, ApJ 517, L23 (1999)

14. L.S. The and S.D.M. White, AJ 92, 1248 (1986)

15. D. Merritt, ApJ 313, 121 (1987)

16. J. Miralda-Escude, ApJ 370, 1 (1991)

17. J.M. Kudo et al, ApJ 671, 1466 (2007)

18. R. Gavazzi et al, A\&A 489, L33 (2009)

19. S.S. McGaugh and J. Wolf, ApJ. 722, 248 (2010)

20. Y. Mellier, G. Mathez, A. Mazura, B. Chauvineau, and D. Proust, A\&A 199, 67 (1988)

21. A. Vikhlinin, W. Forman, and C. Jones, ApJ 435, 162 (1994)

22. P.J.E. Peebles, Principles of Physical Cosmology (Princeton: Princeton University Press) 1993 
23. A.R. Penner, Can. J. Phys. 89, 841 (2011)

\section{Figure Captions:}

Figure 1: Mass density profiles for a) isothermal ICG component, b) stellar component, c) gas localized around NGC 4874 and NGC 4889, d) sum of a), b), and c), e) more concentrated modeled ICG component, f) sum of b), c), and e).

Figure 2: a) baryonic density profile in the case of an isothermal ICG, b) resulting virial density profile in the case of a), c) baryonic density profile in the case of the more concentrated modeled ICG, d) resulting virial density profile in the case of c).

Figure 3: A comparison between the theoretical virial density profiles ( a) for an isothermal ICG and b) for the more concentrated modeled ICG) and the NFW profiles given for the Coma cluster (c) from [18], d) from [17]).

Figure 4: The baryonic mass distributions ( a) for an isothermal ICG and c) for the more concentrated modeled ICG) and the corresponding virial mass distributions (b) corresponding to a) and d) corresponding to c)). Also included are the various virial mass estimates for the Coma cluster that are provide in Section 2: $\mathrm{x}$ - estimates based on X-ray observations, $\mathrm{o}$ - estimates based on velocity dispersion observations, - - estimates based on gravitational shear measurements.

Figure 5: The theoretical shear in the case of a) an isothermal ICG and b) the more concentrated ICG. Included are the shear measurements of [18] $-o$ and [17] - $x$.

Figure 6: Theoretical velocity dispersions for $\beta=0$, corresponding to isotropic orbits, $\beta \rightarrow-\infty$, corresponding to circular orbits, and $\beta=1$, corresponding to radial orbits in the cases of a) an isothermal ICG and b) the more concentrated modeled ICG. Included are the velocity dispersion measurements of $[8]-\mathrm{o}$ and $[15]-\mathrm{x}$ 


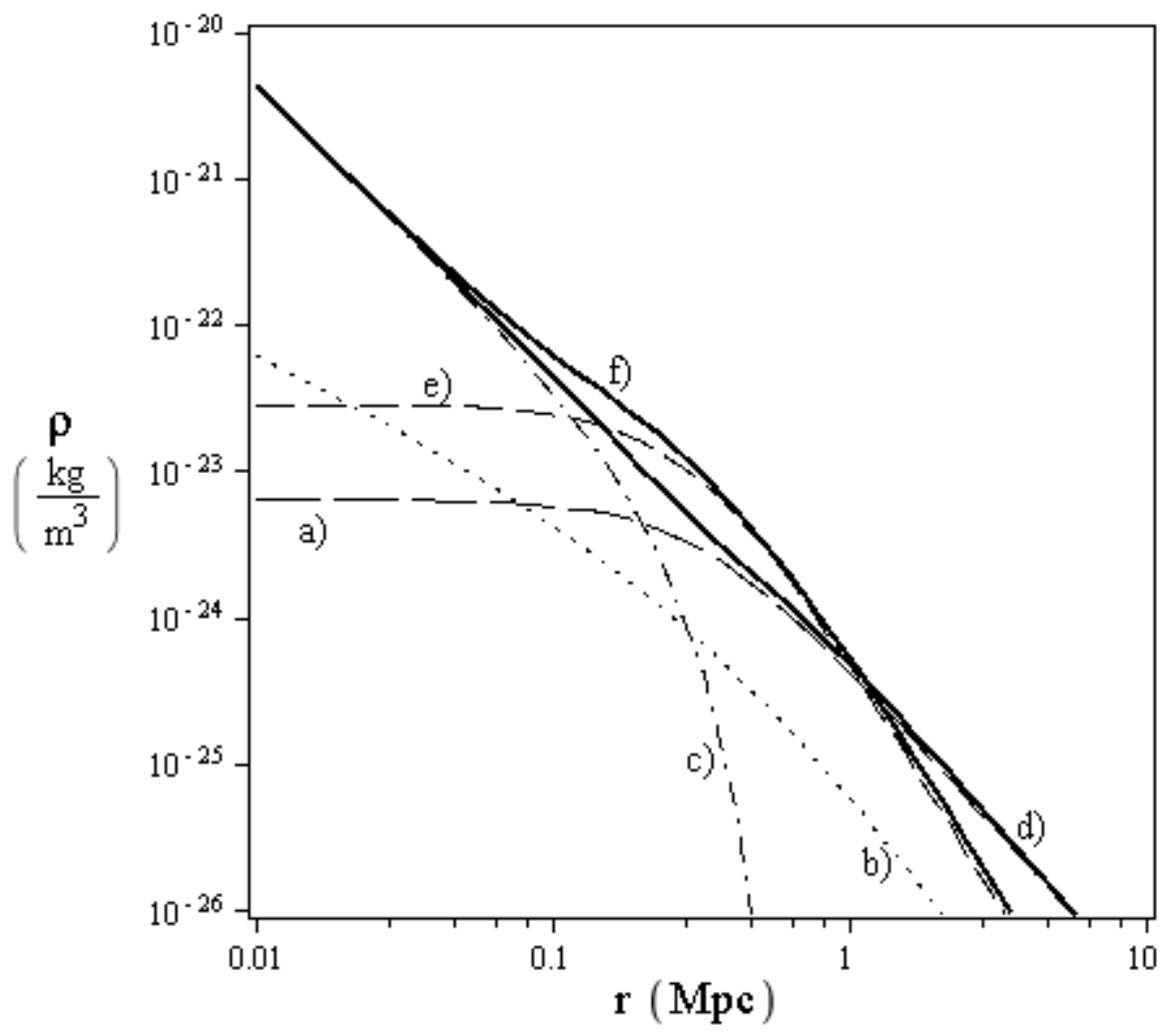

Figure 1 
Penner - Induced energy polarization of the vacuum and the Coma cluster

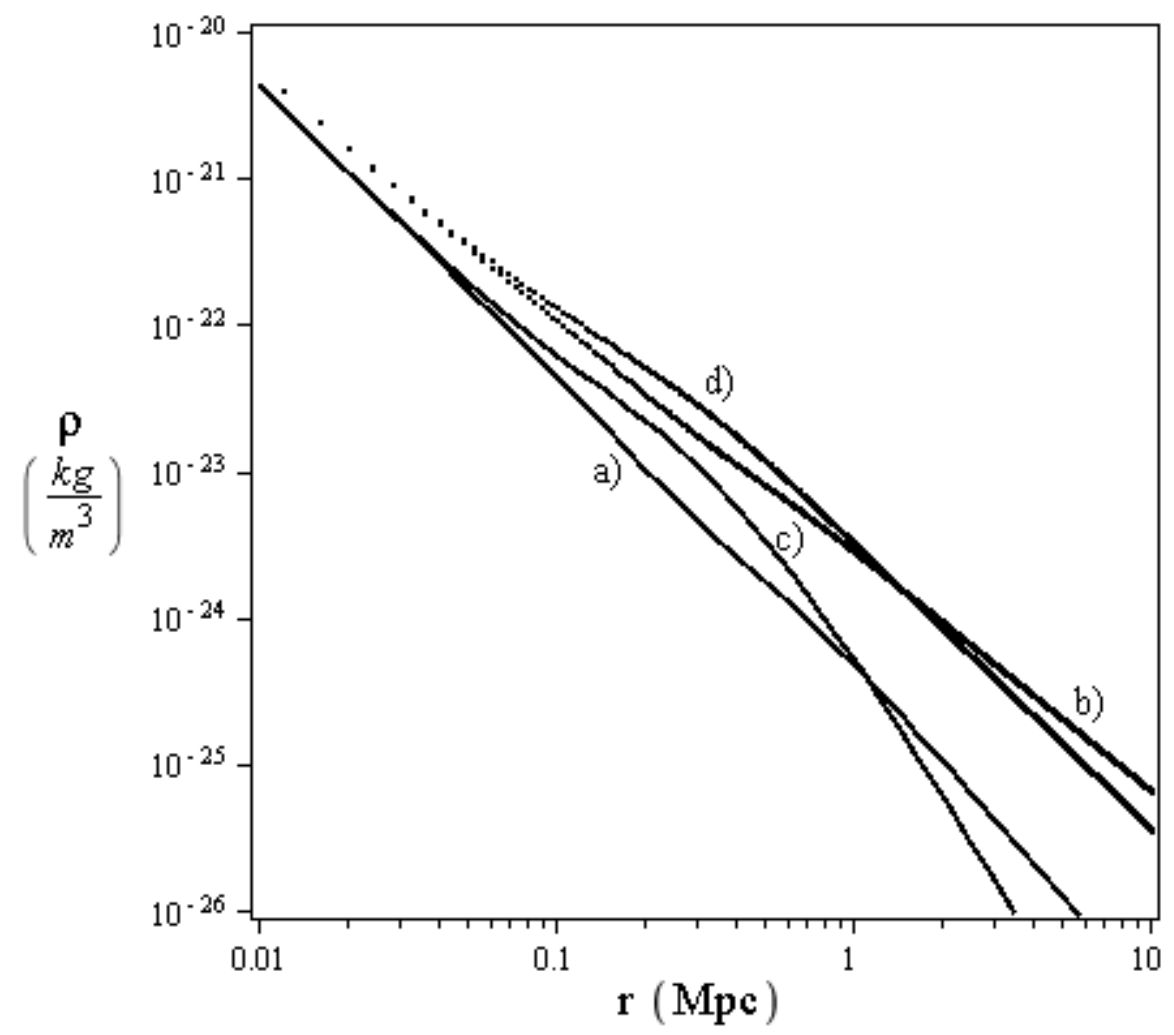

Figure 2 


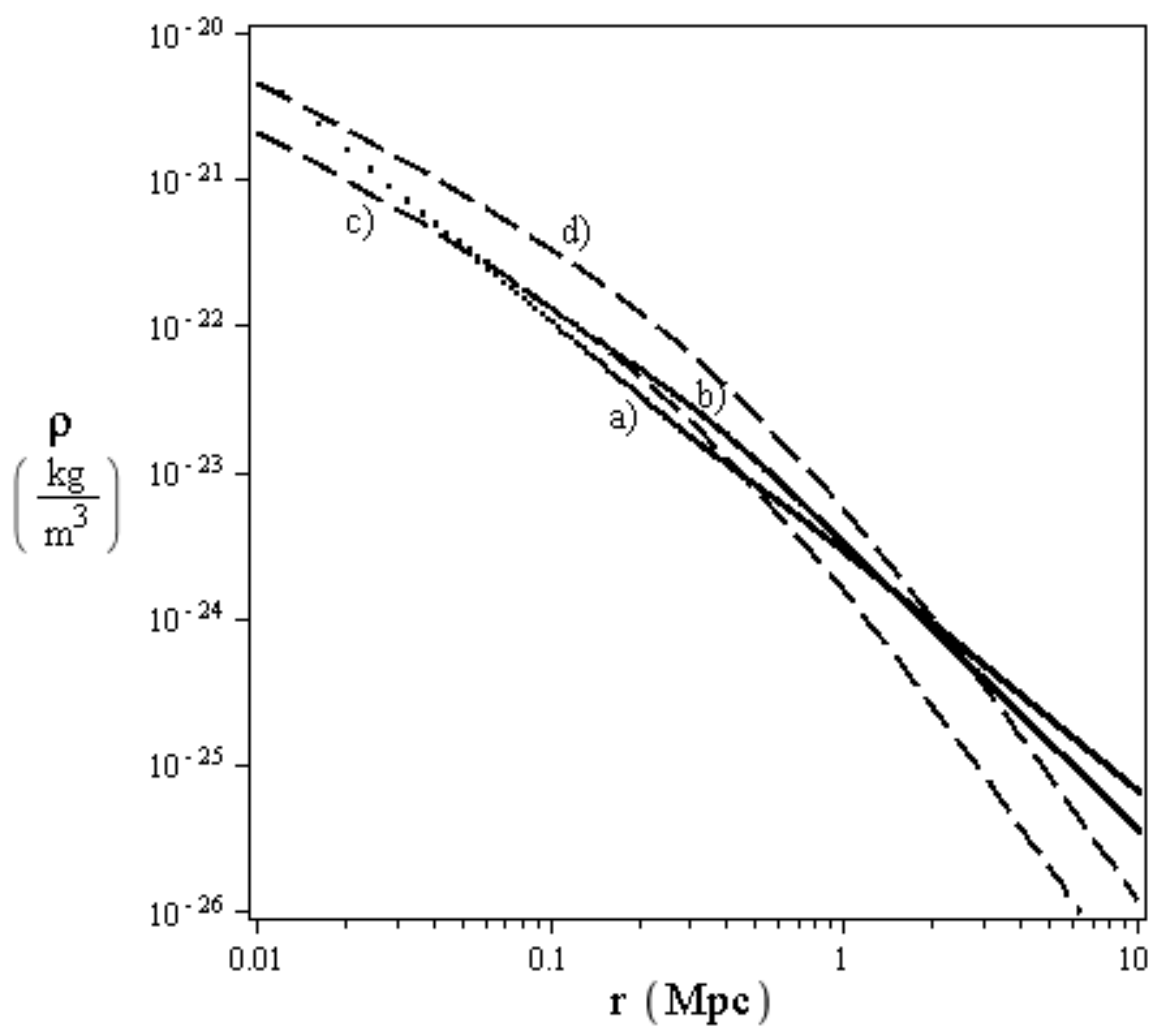

Figure 3 
Penner - Induced energy polarization of the vacuum and the Coma cluster

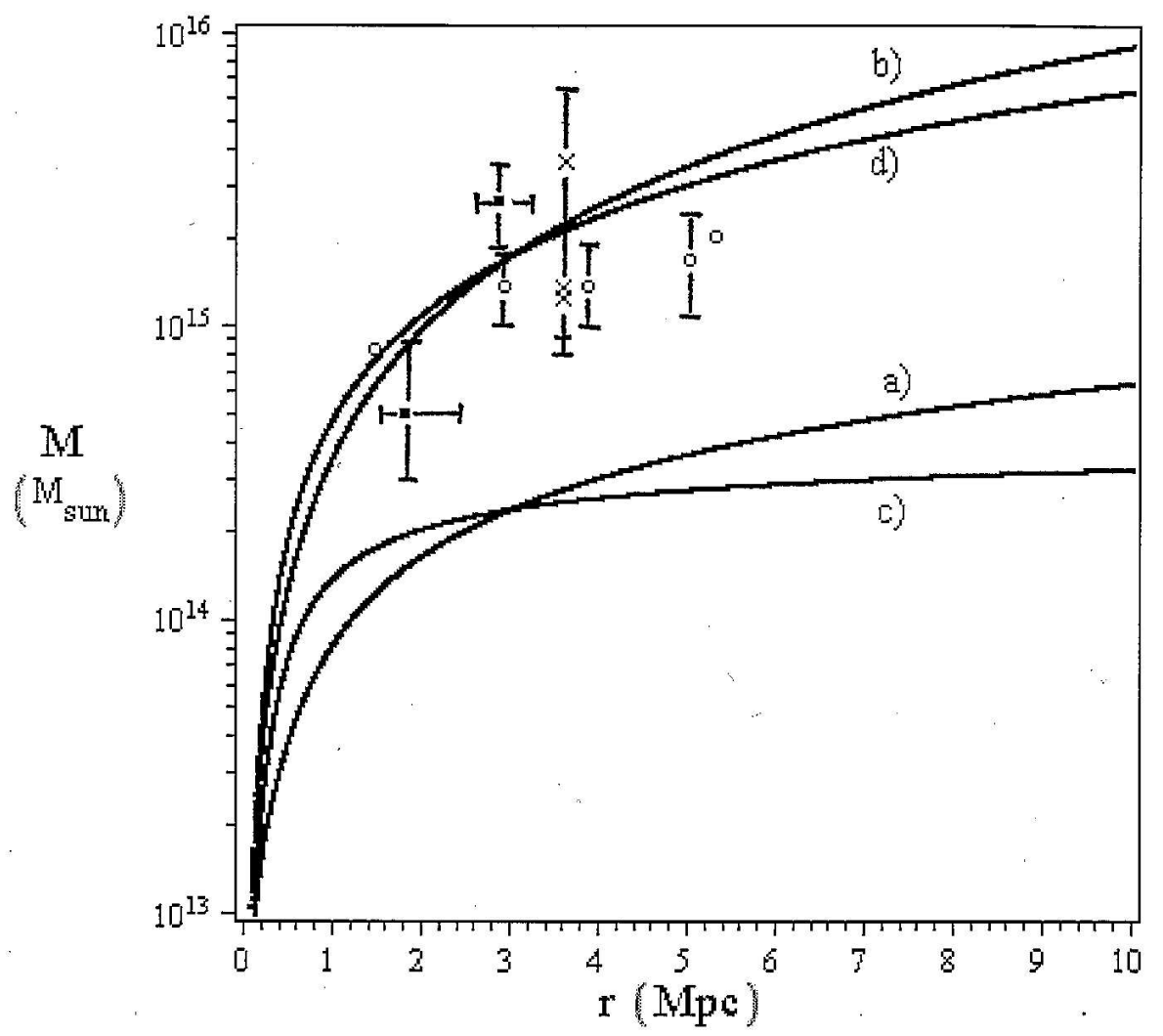

Figure 4 
Penner - Induced energy polarization of the vacuum and the Coma cluster

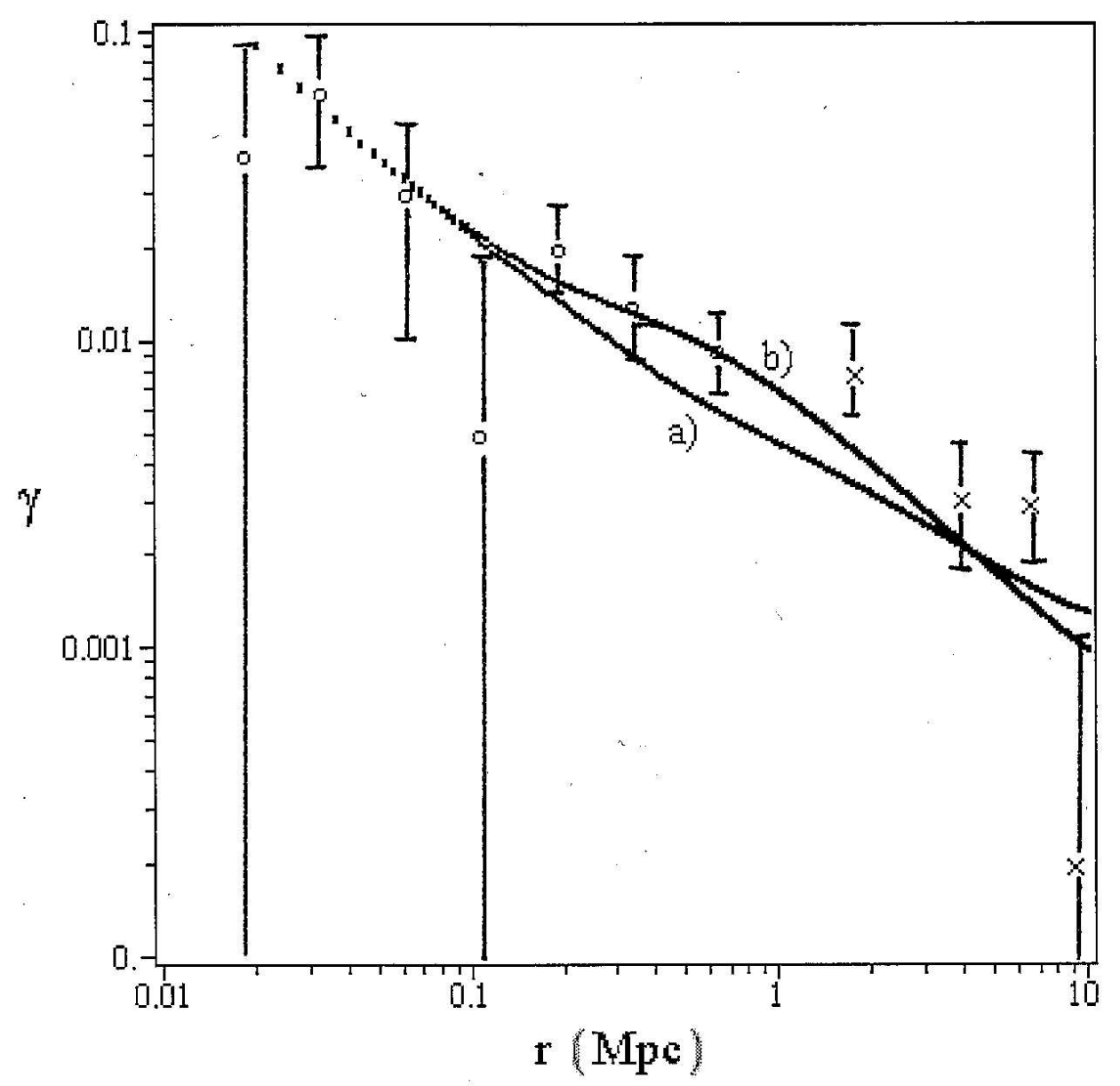

Figure 5 
Penner - Induced energy polarization of the vacuum and the Coma cluster

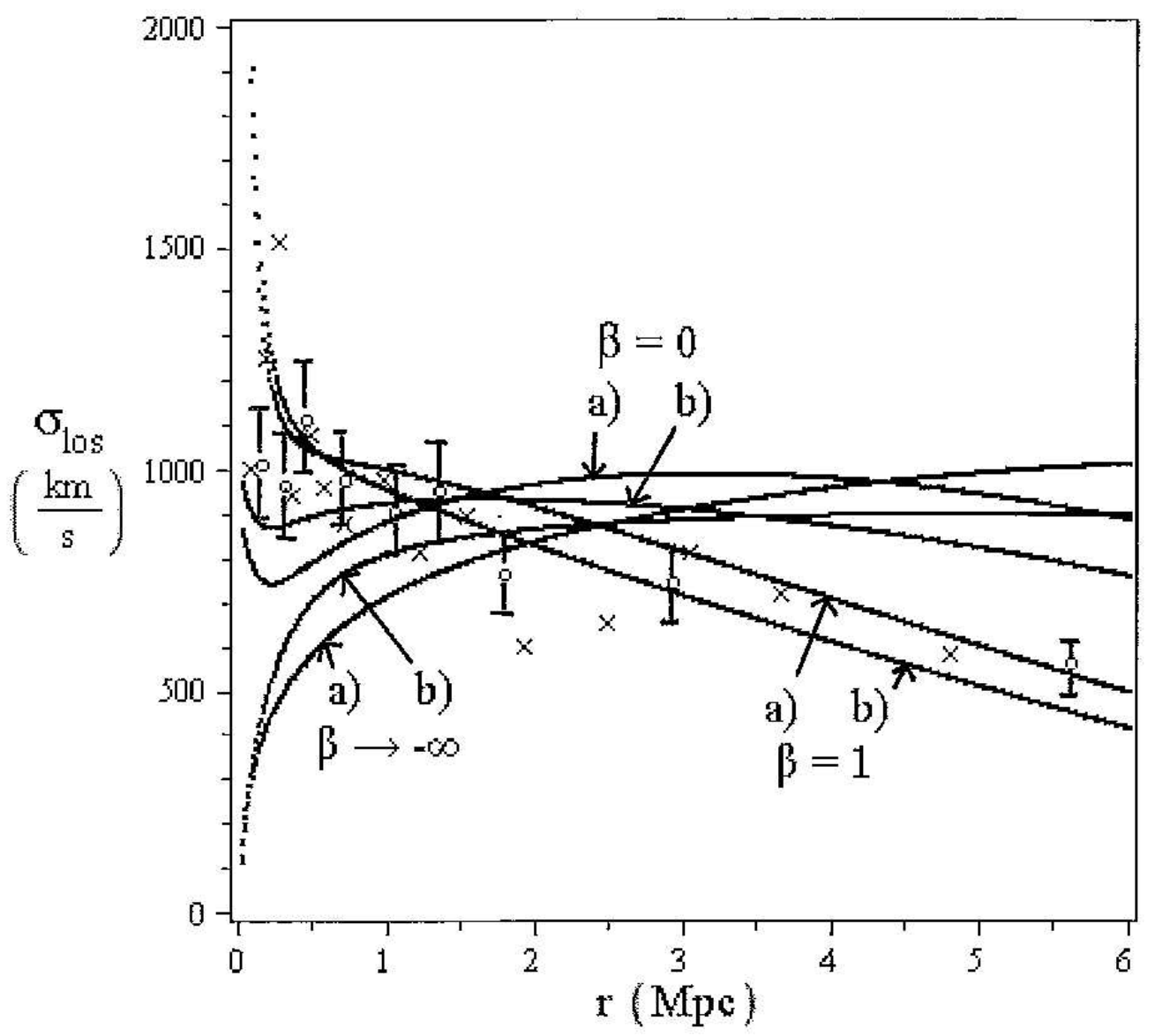

Figure 6 